\title{
Model-based Pose Control of Inflatable Eversion Robot with Variable Stiffness
}

\author{
Ahmad Ataka, Taqi Abrar, Fabrizio Putzu, Hareesh Godaba, and Kaspar Althoefer
}

\begin{abstract}
Plant-inspired inflatable eversion robots with their tip growing behaviour have recently emerged. Because they extend from the tip, eversion robots are particularly suitable for applications that require reaching into remote places through narrow openings. Besides, they can vary their structural stiffness. Despite these essential properties which make the eversion robot a promising candidate for applications involving cluttered environments and tight spaces, controlling their motion especially laterally has not been investigated in depth. In this paper, we present a new approach based on model-based kinematics to control the eversion robot's tip position and orientation. Our control approach is based on Euler-Bernoulli beam theory which takes into account the effect of the internal inflation pressure to model each robot bending segment for various conditions of structural stiffness. We determined the parameters of our bending model by performing a least-square technique based on the pressure-bending data acquired from an experimental study. The model is then used to develop a pose controller for the tip of our eversion robot. Experimental results show that the proposed control strategy is capable of guiding the tip of the eversion robot to reach a desired position and orientation whilst varying its structural stiffness.
\end{abstract}

Index Terms-Modeling, Control, and Learning for Soft Robots; Soft Robot Materials and Design; Motion Control; Eversion Robots; Bio-inspired Robots.

\section{INTRODUCTION}

$\mathbf{I}$ $\mathrm{N}$ the last decade, robots composed of soft and flexible materials have been at the forefront of a new robotics revolution. The use of highly-compliant materials as the main structural element enables these types of robots to perform complex tasks which were not feasible beforehand, employing rigid-component robots. Examples of these new applications include grasping using the whole robot body [1], minimally invasive surgery [2], and navigating in a cluttered and tight space environment [3]. However, one of the challenges faced by the current generation of soft robots is their limited capability in extending their length and in applying high forces to the environment due to their inherently compliant body.

Eversion or vine robots have been emerging recently. Their friction-less advancement along their longitudinal axis has

Manuscript received: September, 10, 2019; Revised December, 17, 2019; Accepted January, 26, 2020.

This paper was recommended for publication by Editor Kyu-Jin Cho upon evaluation of the Associate Editor and Reviewers' comments. This work was supported in part by the EPSRC in the framework of the NCNR (National Centre for Nuclear Robotics) project (EP/R02572X/1) and q-bot led project WormBot (2308/104059).

A. Ataka, T. Abrar, F. Putzu, H. Godaba, K. Althoefer are with the Centre for Advanced Robotics @ Queen Mary (ARQ), Faculty of Science and Engineering, Queen Mary University of London, Mile End Road, London E1 4NS, United Kingdom a.rizqi@qmul.ac.uk

Digital Object Identifier (DOI): see top of this page.

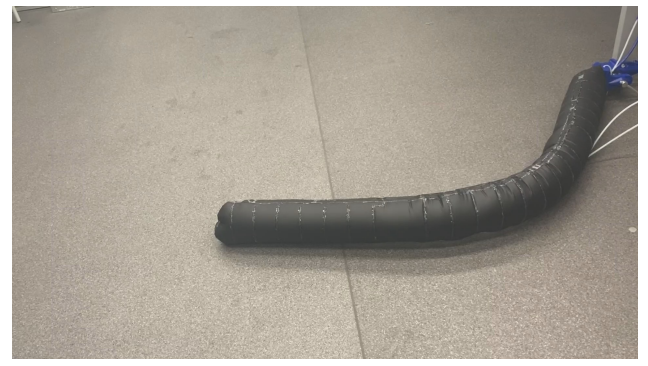

Fig. 1. The soft eversion robot with multiple bending segments.

helped them to establish themselves as excellent candidates to move into remote locations [4], [5]. This type of robot is characterized by its growing-tip movement which, like in a sleeve, turns inside out at the tip, with sleeve material constantly emerging from the inside. Due to this mechanism, the eversion robot can grow from a tiny folded-state into a huge unfolded structure. Applications of robots using the eversion principle include antenna reconfiguration [6], wearable haptic devices [7], soft artificial muscles [8], and soft grippers [9].

Because of its compliance, the eversion robot is able to navigate cluttered environments simply by extending its length and being redirected by the interaction with fixed objects in the environment. Recently, bending capability of inflatable structure has been reported, such as using an antagonistic principle between tendons and pneumatic actuations [10], [11], helically-arranged pneumatically-actuated pouches [12], and longitudinally-arranged pneumatically-actuated pouches [13]. An important characteristic of the fabric-based eversion robot when compared to silicone-based soft robots is the eversion robot's ability to modify its structural stiffness. Hence, an eversion robot is capable of exerting forces on to the environment in a controllable way. Because the outer skin of the eversion robot is made from a non-extensible fabric, forces higher than those that are possible using silicone-based counterparts can be achieved without abandoning the robot's flexibility [8].

Despite these interesting properties which make the eversion robot a promising candidate for applications involving cluttered environments and tight spaces (such as the exploration of an archaeological site [13], laparoscopic surgery [14], nuclear decommissioning, and underfloor exploration), motion control of this type of robot has not been fully investigated. This is mainly caused by the difficulty in modelling the highly nonlinear characteristics of the soft robotic structure in comparison to the well-established modelling of its rigid-link counterparts [15]. Various techniques for the kinematic and dynamic control of soft robotic manipulators have been developed as reported 


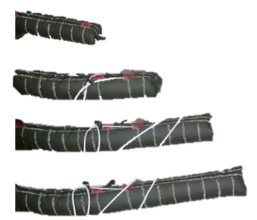

(a)

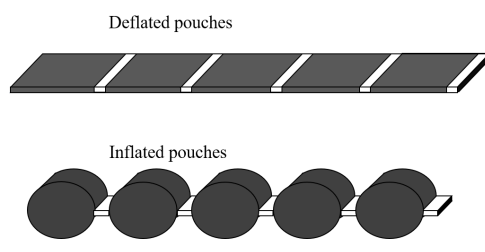

(b)
Fig. 2. (a) A snapshot of our eversion robot during a growing motion. (b) A set of pouches (sewn on the outer skin of the eversion robot) forms a flat surface when deflated and is turned into cylindrical shapes when inflated.

in [16]. Obstacle avoidance for soft or continuum manipulator has also been reported in recent publications [17], [18], [19]. However, none of these works considers the stiffness variation of the robot structure.

Efforts to control an eversion robot were limited only to apical extension [6] and a simple steering mechanism [4]. Recent work tried to perform navigation of an eversion robot by exploiting collisions with nearby obstacles. However, the proposed control mechanism did not involve active steering [20]. Tip position control of an eversion robot has been recently reported in [13], however it relied on a human operator to close the control loop. Orientation control based on visual servoing and position control via growth for eversion robot is reported in [21]. However, stiffness variation which occurs due to the increase in internal pressure once the robot achieves its maximum length is not considered in this work. Recent work explores position and stiffness control of a soft robot with antagonistic pneumatic-tendon actuators [22]. Kinematic control for an inflatable manipulator which considers the change in structural stiffness has also been reported recently [23]. However, none of these works considers a simultaneous position and orientation control of the tip which is crucial in many applications such as object grasping and pick-and-place tasks.

In this paper, we present the first model-based kinematic control of the tip position and orientation of a soft inflatable eversion robot. The proposed controller exploits the unique capability of the inflatable eversion robot to modify its structural stiffness using its inflation pressure. To model the bending section of the robot for various conditions of structural stiffness, Euler-Bernoulli beam theory which takes into account the effect of the internal inflation pressure is employed. The unknown parameters of the model are obtained by performing a least-square method, exploiting the recorded data of the pressure and bending angle retrieved from an experimental study. The model is then exploited to control the tip's position and orientation of a fabric-based eversion robot in a planar environment under varying stiffness condition. To the best of the authors' knowledge, this is the first time modelbased position and orientation control is developed for an inflatable eversion robot with changing structural stiffness.

\section{INFLATABLE EVERSION ROBOT}

One of the recent developments in soft robotics took inspiration from vine plants to achieve growing and stiffening ability

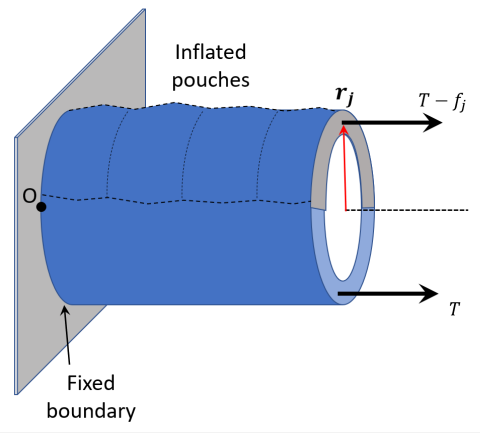

Fig. 3. Model of the inflatable robot with a set of inflated pouches.

in "eversion robot" simultaneously [4], [5]. The structure of this eversion robot (as shown in Fig. 1) is a cylindrical flexible sleeve which is very compact in its folded state. When pressurised, due to the force exerted by air to the structure, the inside of the robot is turned outside, resulting in a longitudinal "growing" motion as shown in Fig. 2a. One of the advantages of this new approach is that the lengthening of the robot occurs only at its tip without affecting the everted body. Moreover, due to the use of non-stretchable fabric as the robot's body, once the robot reaches its maximum length, any increase in the internal pressure will result in variation of the robot's structural stiffness.

To produce bending of the eversion robot, we developed cylindrical pouches integrated with the main structure of the robot. These pouches follow a similar principle to the actuator in [24]: they form a flat surface in a deflated state and they are turned into cylinders in an inflated state, causing the pouches to contract (Fig 2b). The pouches are created by sewing the chosen pattern on two overlapped layers of ripstop fabric. To seal the created seams, a thin layer of natural rubber is applied. Each actuator set is composed of 8 flat rectangles, perpendicularly oriented with respect to the robot's main body. Each bending segment consists of a pair of pouches actuator arranged in antagonistic pairs: activating a set of pouches in one side at a time will cause a difference in length between the pouches on one side and the deflated ones on the other side. This enables us to produce a left or right bending. Having 3 pairs of pouches along the body of the eversion robot enables us to produce multiple independent bending sections as shown in Fig. 1

A set of SMC ITV2050-212L pressure regulators are used to control the air pressure inside the robot's main chamber and inside the pouches. The pressure inside the robot's main chamber will affect the growing motion of the robot as well as its structural stiffness. The pressure inside the pouches will affect the contraction of the pouches which in turn affects the bending angle in each bending segment.

\section{Robot MODEL}

\section{A. Bending model}

Each bending segment of the eversion robot is assumed to behave like an arc of a circle with constant curvature. We assume that each contracting pouches $j$ of a particular bending 
segment produces a tension force $f_{j}$ which is proportional to the given pressure $p_{j}$, i.e. $f_{j} \propto p_{j}$. This tension force produces a bending moment $\mathbf{M}$ with respect to the robot's central axis given by

$$
\mathbf{M}=\sum_{j=1}^{N_{p}} \mathbf{r}_{j} \times \mathbf{f}_{j} .
$$

$\mathbf{r}_{j}$ denotes the vector position of the point where the tension of contracting pouches is applied with respect to the robot's central axis (as shown in Fig. 3) while $N_{p}$ denotes the number of pouches in each bending segment. In this paper, we only consider 2 pouches per bending segment in which only one pouch is activated per bending segment at a time to produce either a left or right bending. Assuming that $f_{j} \propto p_{j}$, the equation can be simplified into

$$
M \approx A p,
$$

where $A$ is a constant while $p=p_{j}$ for the left bending or $p=-p_{j}$ for the right bending.

For simplicity, we assume that the eversion robot follows the formulation for the Euler-Bernoulli beam given by

$$
M=-E I \frac{d^{2} w}{d x^{2}},
$$

where $w(x)$ refers to a beam deflection in a vertical direction at a horizontal position $x$ with respect to the fixed base of the beam, $E$ denotes the structure's Young modulus, and $I$ denotes the cross-sectional moment of inertia. However, as the beam is inflatable, the internal pressure affects the bending rigidity of the eversion robot [25]. The equivalent flexural rigidity for the inflatable beam with axial force $P$ due to the internal inflation pressure and cross-sectional area $S_{0}$ is given by [26]:

$$
(E I)_{e q}=\left(E+P / S_{0}\right) I \text {. }
$$

Compared to the original Euler-Bernoulli formulation in (3), we can see that the flexural stiffness of the overall system is not constant, but rather a function of the axial force $P$ which is proportional to the main chambers pressure $p_{0}$ : the more pressure $p_{0}$ of the main chamber, the stiffer the structure becomes.

The bending angle of a segment of the eversion robot, with respect to the end of the previous segment, caused by the inflation of pouches on one side is obtained by solving the equation $M=-(E I)_{e q} \frac{d^{2} w}{d x^{2}}$, with the zero slope condition $\frac{d w}{d x}=0$. This gives the equation for a small bending angle at a horizontal distance $x$ in the segment of eversion robot as

$$
\theta_{x} \approx \frac{d w}{d x}=\frac{M x}{\left(E+P / S_{0}\right) I} .
$$

Substituting the length of the segment $s$ (i.e. $x \approx s$ for small $\theta_{x}$ ), we obtain the total bending of the specific segment as

$$
\theta_{x} \approx \frac{M s}{\left(E+P / S_{0}\right) I} .
$$

Slotting (1) into (6) and considering the assumption that the parameters $s, S_{0}$, and $I$ are constant and that $P \propto p_{0}$, the bending angle $\theta$ can be simplified into

$$
\theta=\frac{c_{1} p}{1+c_{2} p_{0}}
$$

where $c_{1}$ and $c_{2}$ are positive constant.

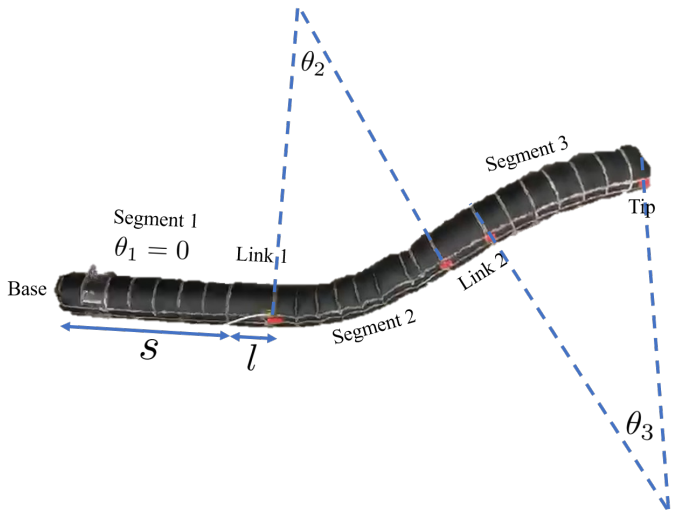

Fig. 4. Eversion robot with 3 bending segments and 2 connecting links.

\section{B. Kinematics model and Jacobian estimation}

A homogeneous transformation matrix describing the pose of the robot's tip can be derived from the bending angle. Suppose that we have $N$ number of bending sections. The actuator space variable $\mathbf{p}_{a c t}=\left[\begin{array}{ll}p_{0} & \mathbf{p}\end{array}\right]^{T}$ consists of pressure for the main chamber $p_{0}$ and pouches' pressure for all bending segments $\mathbf{p}$. The pouches' pressure is defined as $\mathbf{p}=\left[\begin{array}{lll}p_{1} & \ldots & p_{N}\end{array}\right]^{T}$ where $p_{i}$ is pressure in bending segment$i$. The overall bending angle variables are described by $\Theta=\left[\begin{array}{llll}\theta_{1} & \theta_{2} & \ldots & \theta_{N}\end{array}\right]^{T}$. Assuming that each bending section satisfies a constant curvature condition, the homogeneous transformation matrix of the end section with respect to the base for bending section- $i, \mathbf{T}_{i}^{b} \in S E(2)$, can be described as

$$
\mathbf{T}_{i}^{b}=\left[\begin{array}{ccc}
\cos \theta_{i} & -\sin \theta_{i} & \frac{\left(1-\cos \theta_{i}\right)}{\theta_{i}} s \\
\sin \theta_{i} & \cos \theta_{i} & \frac{\sin \theta_{i}}{\theta_{i}} s \\
0 & 0 & 1
\end{array}\right] .
$$

However, eq. (8) can lead to a kinematic singularity during the zero bending condition $\left(\theta_{i}=0\right)$. To avoid this, the last column of $\mathbf{T}_{i}^{b},\left[\mathbf{T}_{i}^{b}\right]_{3}$, is modified into its limit value when $\theta_{i}=0$, i.e. $\left[\mathbf{T}_{i}^{b}\right]_{3}=\lim _{\theta_{i} \rightarrow 0}\left[\begin{array}{lll}\frac{\left(1-\cos \theta_{i}\right)}{\theta_{i}} s & \frac{\sin \theta_{i}}{\theta_{i}} s & 1\end{array}\right]^{T}=\left[\begin{array}{lll}0 & s & 1\end{array}\right]^{T}$.

Except for the most distal section, we have a connecting link with an identical length of $l$ after every bending section as illustrated in Fig. 4 for the case of $N=3$. Suppose that the homogeneous transformation matrix describing the endpoint of the link with respect to its base is given by $\mathbf{T}^{l} \in S E(2)$, then, the homogeneous transformation matrix of the robot's tip with respect to the robot's base is

$$
\mathbf{T}^{t}=\left[\begin{array}{cc}
\mathbf{R} & \mathbf{x} \\
\mathbf{0} & 1
\end{array}\right]=\left(\prod_{i=1}^{N-1} \mathbf{T}_{i}^{b} \mathbf{T}^{l}\right) \mathbf{T}_{N}^{b} .
$$

Note that combining (7) and $(9)$ results in a forward kinematic mapping from the actuator space $\mathbf{p}_{a c t}$ to the task-space position $\mathbf{x} \in \mathbb{R}^{3}$ and orientation $\theta^{t}$ which can be derived from a rotational matrix $\mathbf{R} \in S O(2)$ in $(9)$ or, due to the planar configuration, can be written as $\theta^{t}=\sum_{i=1}^{N} \theta_{i}$. Exploiting the forward kinematics relation in 99, one can then derive the positional Jacobian $\mathbf{J}^{p}=\frac{\partial \mathbf{x}}{\partial \mathbf{p}} \in \mathbb{R}^{2 \times N}$ and orientation Jacobian $\mathbf{J}^{o}=\frac{\partial \theta^{\mathbf{t}}}{\partial \mathbf{p}} \in \mathbb{R}^{1 \times N}$ numerically. Note that these Jacobian matrices are singularity-free due to the use of limit values to modify 


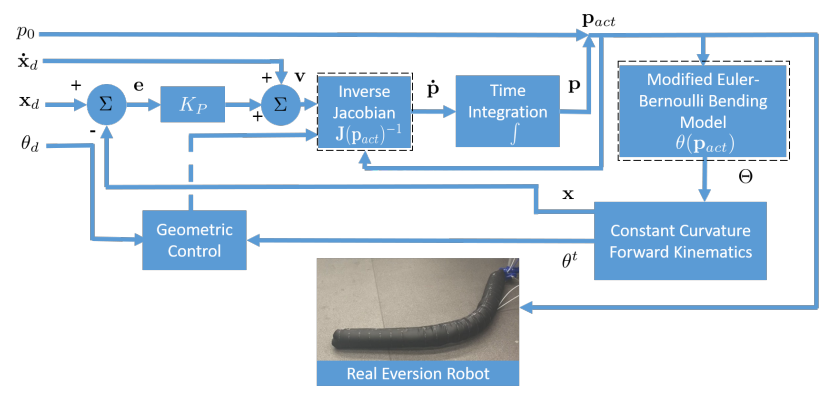

Fig. 5. The block diagram of the proposed model-based control of eversion robot in a sensor-less scenario. The blocks inside dashed squares use parameters $c_{1}$ and $c_{2}$ retrieved from the system identification experiment.

the original eq. (8) when $\theta_{i}=0$. Note that only the pressure of the pouches $\mathbf{p}$ is used to calculate the Jacobian. The pressure of the main chamber $p_{0}$ is excluded since it will not be a part of the control signal as will be explained further in the next section.

\section{MOdEL-BASEd CONTROL}

In this section, we describe the control policy used to guide the tip's pose towards the desired target for varying conditions of the robot's structural stiffness. The pressure in each of the contracting pouches $p_{i}$ used to create the bending is produced by the controller at every iteration. The internal inflation pressure $p_{0}$, however, is controlled directly by the user during the robot's movement to modify the robot's structural stiffness. This characteristic is useful for applications where the robot is expected to modify its structural stiffness on-demand during the performance of a task. The variation of this internal pressure will be taken into account in the control loop to ensure that the task can still be completed under the variation of structural stiffness. We assume that no external sensor is available to detect the shape of the eversion robot when it is moving. This scenario is useful in applications where the use of an external sensor such as camera is not feasible.

The block diagram of this approach is shown in Fig. 5. From the set of pressure values produced by the pressure regulators $\mathbf{p}_{a c t}$, we get the tip's position $\mathbf{x}$ and orientation $\theta^{t}$ from 9 . To reach a desired tip position $\mathbf{x}_{d}$, a proportional control is used in the robot's task-space as follows

$$
\dot{\mathbf{x}}=-K_{P}\left(\mathbf{x}-\mathbf{x}_{d}\right),
$$

where $K_{P}$ stands for a positive constant. For a tracking task, where the robot's tip needs to track a point moving on a trajectory $\mathbf{x}_{d}(t)$, the following control law is used in the taskspace

$$
\dot{\mathbf{x}}=-K_{P}\left(\mathbf{x}-\mathbf{x}_{d}\right)+\dot{\mathbf{x}}_{d} .
$$

To control the orientation, a geometric-based control based on [27] is employed. Suppose that the tip's orientation $\theta^{t}$ is described by a rotation matrix $\mathbf{R} \in S O(2)$ while the desired orientation $\theta_{d}$ is described by $\mathbf{R}_{d} \in S O(2)$. By introducing an error matrix $\mathbf{R}_{e}=\mathbf{R}_{d}^{T} \mathbf{R}$, the following control law is used in the task-space

$$
\hat{\omega}=-K_{\omega} \log \left(\mathbf{R}_{e}\right)
$$

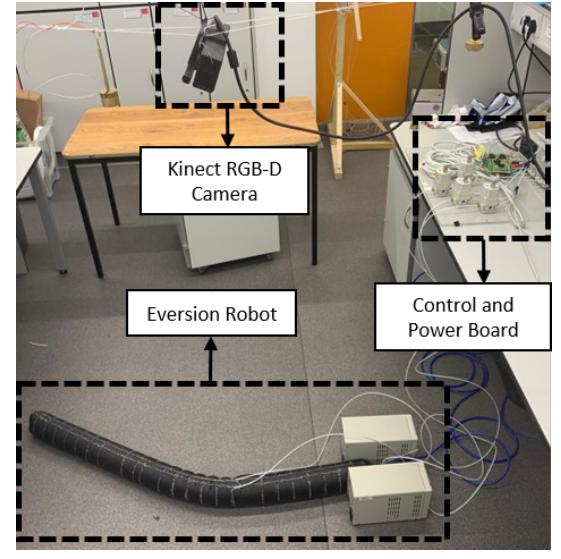

Fig. 6. The experimental setup for system identification process.

where $K_{\omega}$ refers to a positive constant and the operator $\log (\mathbf{R})$ for any $\mathbf{R} \in S O(2)$ is defined as $\log (\mathbf{R})=\frac{\beta}{2 \sin \beta}\left(\mathbf{R}-\mathbf{R}^{T}\right)$ where $\beta=\arccos \left(\frac{\operatorname{tr}(\mathbf{R})}{2}\right)$. The angular speed $\omega \in \mathbb{R}^{3}$ can be retrieved from a skew-symmetric matrix $\hat{\omega}$. Finally, considering the planar environment, the angular velocity $\dot{\theta}$ is a $z$-component of $\omega$.

To transform the task-space velocity $\mathbf{v}$ into an actuator-space velocity $\dot{\mathbf{p}}$, we use the resolved-rate control as follows [28]

$$
\dot{\mathbf{p}}=\left\{\begin{array}{ll}
\mathbf{J}^{+} \mathbf{v}, & \text { if } \mathbf{J} \text { is a non-square matrix } \\
\mathbf{J}^{-1} \mathbf{v} & \text { if } \mathbf{J} \text { is a square matrix }
\end{array},\right.
$$

where $\mathbf{A}^{+}=\mathbf{A}^{T}\left(\mathbf{A} \mathbf{A}^{T}\right)^{-1}$. For control of position without orientation, the task-space velocity $\mathbf{v}$ consists of a linear velocity $\dot{\mathbf{x}}$ in (10) or (11) while the Jacobian is given by $\mathbf{J} \in \mathbb{R}^{2 \times N}=\mathbf{J}^{p}$. For simultaneous position-orientation control, the task-space velocity $\mathbf{v}$ consists of a linear velocity $\dot{\mathbf{x}}$ and an angular velocity $\dot{\theta}$ derived from 12 while the Jacobian is given by $\mathbf{J} \in \mathbb{R}^{3 \times N}=\left[\begin{array}{ll}\mathbf{J}^{p} & \mathbf{J}^{o}\end{array}\right]^{T}$.

\section{RESUlts AND ANALYSIS}

\section{A. System Identification}

Before implementing the control policy for the eversion robot, a system identification process is performed to get the parameters of the bending model described in (7). The setup of the modelling experiment is shown in Fig. 6 In this process, different combinations of input pressure values are given to each bending sections of the robot. Every time a set of pressure is given, an 8-seconds time delay is given for the pouches to reach the bending angle. The resulting bending angle is then retrieved from the image of the robot captured using a camera fixed on top of the robot. The main chamber pressure $p_{0}$ ranges from 0.3 bar to 1.2 bar with 0.05 bar step. The pressure which is given to the pouch of bending section $p_{i}$ ranges from 0 to 1.5 bar with 0.05 bar step for both the left and right bending direction. A total of 1177 combinations of pressure values is used to test each segment as shown in Fig. 7a. The total duration of the experiment for each bending segment is, therefore, $1177 \times 8$ seconds $=9416$ seconds. To get the bending angle, markers with a distinct colour are put in several locations on the robot body. From the location of 2 


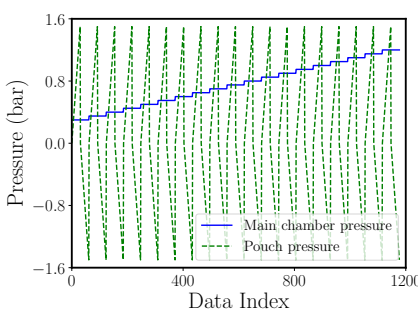

(a)

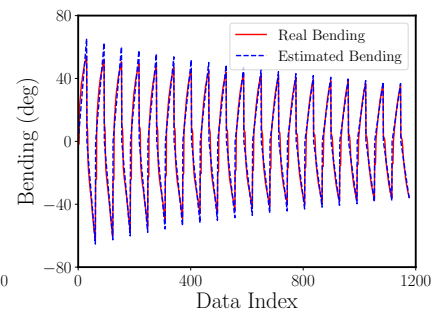

(b)

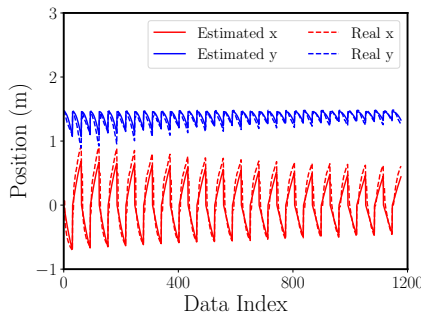

(c)

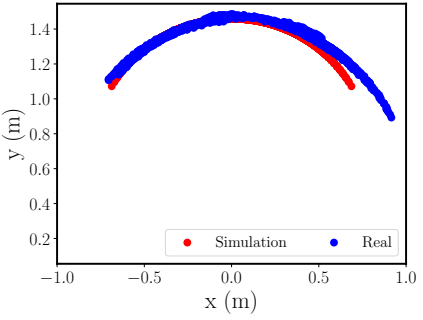

(d)

Fig. 7. (a) The pressure given to the robot during the system identification which consists of the main chamber's pressure and the pouch's pressure for bending. (b) The bending angle from the experiment and the estimated bending angle produced by the model. (c) The comparison between the real (dashed line) and the predicted (solid line) tip's position. (d) Comparison between the real (blue dots) and the predicted (red dots) tip's position.

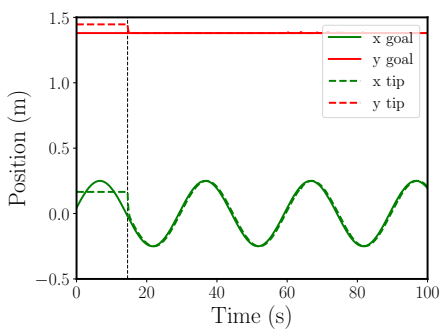

(a)

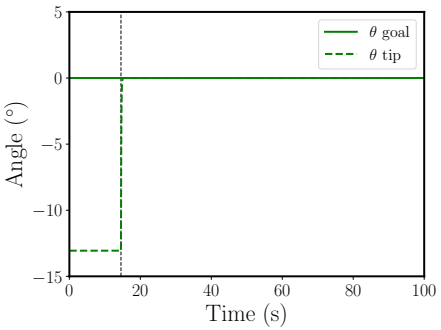

(b)

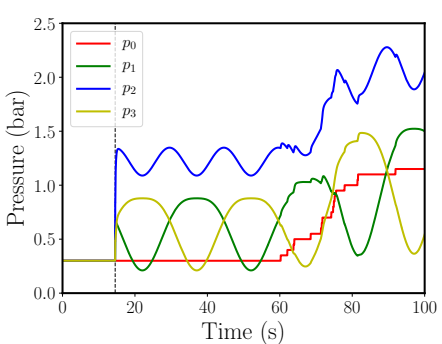

(c)

Fig. 8. The simulation results of the position and orientation control task for a robot with 3 bending segments $(N=3$ ) show the plot of (a) the tip's (dashed lines) and goal position (solid lines), (b) the tip's (dashed lines) and goal orientation angle (solid lines), and (c) the input pressure values given to the main chamber (red line) and each bending pouches (green, blue, and yellow respectively). The dashed vertical line is the time when the robot starts to perform trajectory tracking.

most distal markers in the pixel space of the recorded images, we get a straight line equation from which a bending angle $\theta$ with respect to the vertical line (a condition where $\theta=0$ ) can be retrieved. Finally, we get a data set consisting of the main chamber's pressure $p_{0}$, the bending pressure $p_{i}$, and the bending angle $\theta$ at every step. Curve fitting based on leastsquare optimization is employed to fit the recorded data with the model described in (7). From this process, the parameters $c_{1}$ and $c_{2}$ in (7) are retrieved for each bending segment.

Fig. $7 \mathrm{~b}$ shows the comparison between the estimated bending angle (plotted in a dashed blue line) produced by the model using these parameters and the real bending angle retrieved from the experiment (plotted in a red line) for the 1177 combinations of pressure values shown in Fig. 7a We can see that the predicted bending angle has a very similar value to the recorded bending angle, especially for the case of small bending angle. Bigger error occurs in the case of bigger angle due to the approximation used in the bending model, particularly the linear assumption in the actuators' response described in (2) and in the structure's response described in (3). However, the model in (7) can still predict the bending angle with a root-mean-square error of $6.88^{\circ}$.

In Fig. 7c-Fig. 7d, we can observe how the kinematic model which predicts the tip's position compares with the experimental data. In Fig. 7c we can see that the predicted tip's position shown as a red solid line for $x$ axis and blue solid line for $y$ axis matches closely with the real tip's position shown as a red dashed line for $x$ axis and blue dashed line for $y$ axis for the given input signal. This performance is also reflected in Fig. 7d where the predicted position of the tip in $x y$ plane shown as a red dot closely matches the real position of the tip shown as a blue dot. This confirms the validity of the constant curvature assumption in (8)-(9).

\section{B. Simulation Results}

The bending model retrieved from the system identification experiment is used in this section to implement the modelbased control as described in Section IV] Prior to implementing the controller to the real eversion robot, we test the performance of the controller under a variation of the robot's structural stiffness in a simulation scenario. The parameters of the robot and the controller are $s=0.42 \mathrm{~m}, l=0.1 \mathrm{~m}$, $K_{P}=K_{\omega}=5$. The control algorithm is running at $50 \mathrm{~Hz}$ frequency.

Fig. 8 shows the performance of the model-based position and orientation control when it is applied to the model of an eversion robot. The task is to track a trajectory $\mathbf{x}_{d}(t)$ moving back and forth in a straight line. At the same time, the tip of the robot also needs to stay at the desired orientation angle $\theta_{d}=0$. While executing the task, the structural stiffness of the robot's body will be modified by changing the pressure of the main chamber $p_{0}$.

In Fig. 8a, we can see that the robot's tip, shown in dashed green $(x)$ and red $(y)$ lines, starts from an arbitrary location. Then, the robot starts tracking the goal position at $t=15 \mathrm{~s}$, indicated by a dashed black vertical line. We can observe that the controller is able to ensure the robot's tip to follow the 


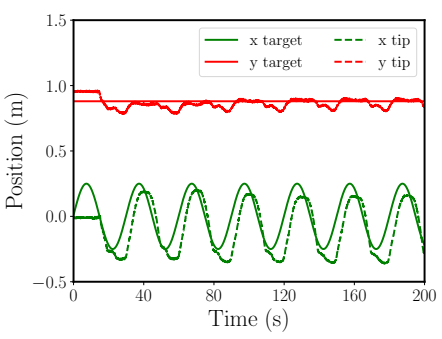

(a)

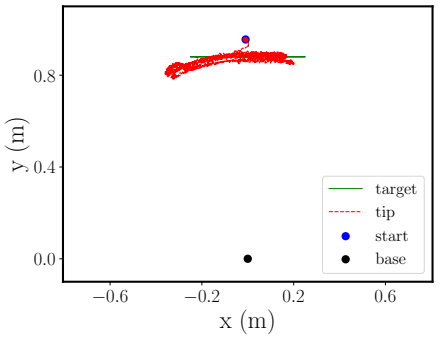

(b)

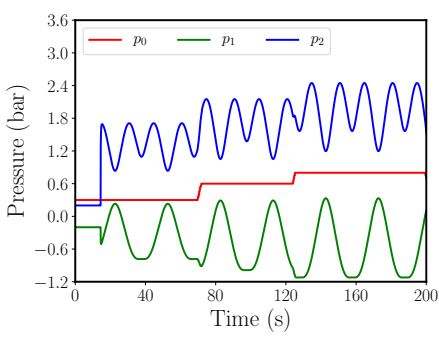

(c)

Fig. 9. The experimental results of the position (without orientation) control task for a robot with 2 bending segments $(N=2)$ show the plot of (a) the tip's (dashed lines) and goal position (solid lines) as a function of time, (b) the tip's trajectory (red dashed lines) and the desired trajectory (green solid lines) in the $x y$-plane, and (c) the input pressure values given to the main chamber (red line) and each bending pouches (green and blue respectively).

desired trajectory shown as solid green and red lines. At the same time, in Fig. 8b, we can also observe that the controller guides the tip to reach the desired orientation while tracking the trajectory. At $t=60 \mathrm{~s}$, we modify the pressure of the main chamber $p_{0}$, shown as a red line in Fig. 8c, which will modify the robot's structural stiffness. However, the trajectory tracking performance is not affected as shown by a stable tracking for the whole movement in Fig. 8a and Fig. 8b This performance is achieved since the model, which is described in (7) and retrieved from the system identification process in Section $\mathrm{V}-\mathrm{A}$, considers the variation of the main chamber's pressure. Henceforth, the controller is able to compensate a higher stiffness state by producing more pressure for the bending pouches. This can be observed in Fig. $8 \mathrm{c}$ when the bending pressures $p_{1}, p_{2}$, and $p_{3}$ (depicted by the green, blue, and yellow lines respectively) get bigger to maintain the tracking performance of the tip in a higher stiffness condition.

\section{Experimental Results}

We implement the proposed controller in the real eversion robot. To evaluate the performance of the controller, the real tip's position $\mathbf{x}$ and orientation $\theta^{t}$ of the robot are retrieved from the RGB-D Camera mounted in a configuration shown in Fig. 6 . An image processing technique is used to detect the markers on the robot body. The location of the most distal marker with respect to the base marker reflects the tip position $\mathbf{x}$ in the pixel space which can be transformed into metres using a weight $W$ retrieved from an initial camera calibration. From the location of 2 markers on the most distal bending section and 2 markers on the first bending section closest to the base, we can get two line equations from which the tip's bending angle $\theta^{t}$ can be retrieved.

We start by using only 2 bending sections $(N=2)$ and implementing only position control to track a straight line trajectory similar to the simulation scenario without the orientation control. The complete results are shown in Fig. 9 . In Fig. 9a, we can see that the robot's tip (whose motion is shown in dashed lines) starts from an arbitrary position before tracking the desired trajectory $\mathbf{x}_{d}(t)$ (shown in solid lines). The movement is not as smooth as the simulation results, however, we can observe that the tracking task is still achieved. There is a notable delay in the tracking process which can be observed especially in the graph of the position in $x$ axis (green lines).

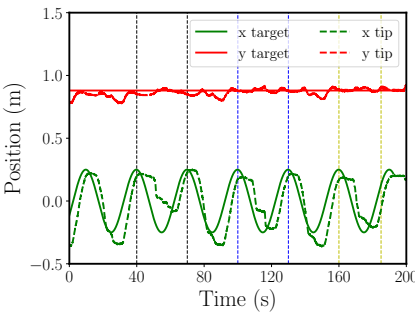

(a)

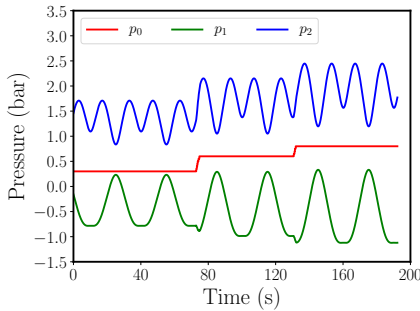

(b)
Fig. 10. The position tracking task for a robot with 2 bending segments $(N=2)$ which includes an interaction with an object shows the plot of (a) the tip's (dashed lines) and goal position (solid lines) and (b) the input pressure values given to the main chamber (red line) and each bending pouches (green and blue). The pairs of vertical lines with the same colour indicate the time windows when an object is put on the path of the robot.

This is caused by the fact that the air takes time to go inside and fully fill the pouches when producing the bending angle. The observed time delay can reach up to approximately 5 seconds, depending on the changes of bending angle during the movement. This phenomenon is not considered in the current bending model, however, the tracking task is still achieved. The tip's position (red dashed line) and the desired trajectory (solid green line) are also plotted in the $x y$-plane as can be observed in Fig. 9b Once again, we can observe that the tracking performance is maintained despite the variation of the internal pressure $p_{0}$ shown as a red line in Fig. $9 \mathrm{c}$ which in turns affects the robot's stiffness. This performance is achieved since the model-based control compensates the higher stiffness condition with a bigger pressure sent to the pouches (depicted by green and blue lines) to maintain the task execution.

In the next set of experiment, we demonstrate the usefulness of stiffness variation. When executing a task, such as trajectory tracking, in a cluttered environment, the manipulator could be obstructed by objects. In this experiment, we will show that having stiffness-controllability enables the inflatable robot to perform a trajectory tracking task while having an ability to change its stiffness to push away an obstruction on its path. For this purpose, an object is placed on the path of the robot at a certain time during the tracking movement. The full results are shown in Fig. 10

In Fig. 10a at $t=0 s$, the robot starts to track the moving target. At this time, we set the internal pressure $p_{0}$ to be small 


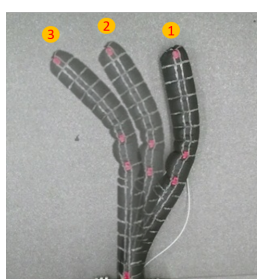

(a)

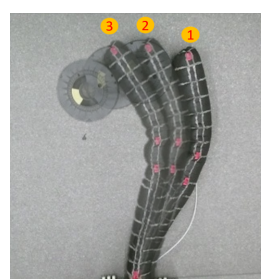

(b)

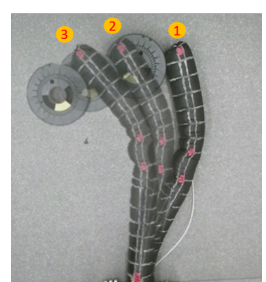

(c)
Fig. 11. The snapshots of the robot during the trajectory tracking (a) when there is no object obstructing the robot, (b) when there is an object for an internal pressure of $0.3 \mathrm{bar}$, and (c) when there is an object for an internal pressure of 0.7 bar. The order of the movement is from a shape labelled 1 , 2 , and finally 3 .

which can be observed in Fig. 10b (red line). This causes the robot's structural stiffness to be low and, as a result, the controller does not need high pressure for the pouches to create the bending movement as reflected by a relatively lower pressure $p_{1}$ and $p_{2}$ in Fig. 10b At $t=38 s$, when the first black vertical line is placed in Fig. 10a, an object is placed on the path of the robot. Due to the low structural stiffness, the robot is not able to apply a high force during the contact with the object, so the robot's tip is not able to continue following the target trajectory. This is shown by a large deviation between the desired $x$ position (solid green line) and the real $x$ position of the tip (dashed green line) in Fig. 10a in between the 2 black vertical lines. After the second black vertical line $(t>70 s)$, the object is removed and, at the same time, the internal pressure $p_{0}$ is increased to make the stiffness higher shown as a red line in Fig. 10b. Once again, the robot is able to track the moving target. At $t=100$ s (the first blue vertical line), the same object is placed on the path of the robot. This time, due to the higher structural stiffness it possesses, the robot is able to move the object to maintain the tracking task as much as possible. This is reflected by better tracking of the robot's tip position in $x$ direction (dashed green line) with respect to the target's position in $x$ direction (solid green line) in Fig. 10a in between the 2 blue vertical lines compared to the previous scenario. Afterwards, a similar set of scenario is tested in a period between the 2 yellow vertical lines in Fig. 10a for even higher stiffness which yields a similar result. The snapshots of these scenarios can also be observed in Fig. 11 where we compare the performance of the trajectory tracking when there is no object obstructing the robot (Fig. 11a), when there is an object for a low internal pressure $p_{0}=0.3$ bar (Fig. 11b), and for a high internal pressure $p_{0}=0.7$ bar (Fig. [11c).

The experimental results in Fig. 10 and Fig. 11 confirm the usefulness of stiffness variation which enables the robot to produce a higher contact force when interacting with an object. It is noted however that the controller in its current state cannot guarantee that the tracking error goes to zero in the presence of the external force such as the case when the robot is in contact with the environment. This is the case since the bending model described in Section [II] does not yet consider the effect of the external force on the robot's bending model.

The final experimental scenario deals with a position and orientation tracking for an eversion robot with 3 bending sections $(N=3)$. We set a fixed goal position for the robot's tip and a sinusoidal function as a desired orientation angle of the tip. The complete results are shown in Fig. 12. In Fig. 12a, we can see that the proposed controller is able to keep the robot's tip to stay at a fixed position in $x$ (dashed green line) and $y$ direction (dashed red line) with slight fluctuation. There is, however, a notable deviation from the desired position (green and red solid lines). A similar phenomenon is observed in Fig. $12 \mathrm{~b}$ where we can observe that the proposed controller enables the robot's tip to follow a sinusoidal orientation angle $\theta^{t}$ despite there is a slight deviation from the desired orientation angle $\theta_{d}(t)$. A possible reason for this is the fact that the kinematic model employed does not consider the effect of friction between the robot's body and the floor. Thus, when the desired task necessitates the robot to perform a minimal movement, such as the case for this task, the resulting action force due to the pressure signal produced by the controller is not significant enough to work against static friction between the robot and the floor.

The problem of model inaccuracy can be solved by employing a more accurate model which considers static and kinetic friction between the robot body and the environment. Another solution is to employ an additional sensor, such as a camera or bending sensor, as feedback in the control loop. In the presence of a visual feedback, for instance, a visual servoing technique can be employed to compensate for model inaccuracy. Despite this limitation, the results described in this section demonstrate that the proposed model-based control can still achieve a good performance to control the pose of the inflatable eversion robot for various conditions of structural stiffness even without sensors to close the loop. This is useful for applications where the use of an external sensor is not feasible, such as nucleardecommissioning tasks or an underfloor exploration. A future study will explore the use of on-board sensors which can be integrated into the fabric, such as flexible bending sensor, to help to improve the estimation of the robot's states, and hence, improving the overall performance of the controller.

\section{CONClusions AND Future Works}

In this paper, we develop a model-based kinematic control approach for a soft, inflatable, eversion robot. An EulerBernoulli beam theory is used to model the bending section of the robot which takes into account the effect of the internal pressure to the structural stiffness of the robot. The unknown parameters of the model are retrieved by exploiting the recorded data of the pressure and bending angle from an experimental study. The model is then used for position and orientation control of the tip of the eversion robot. We show that the proposed method is able to guide the robot's tip to follow a trajectory in a 2-dimensional space. The tracking performance is achieved despite variation of the robot's internal inflation pressure which affects the robot's structural stiffness. Future work will explore the use of a more advanced model, such as a static model which considers friction or a dynamic model of the robot, and a more advanced control algorithm. The effect of the external force to the robot's model will be explored to handle situations where the robot needs to 


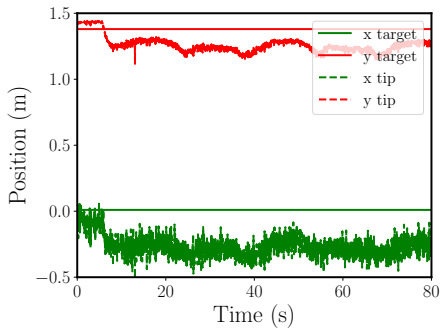

(a)

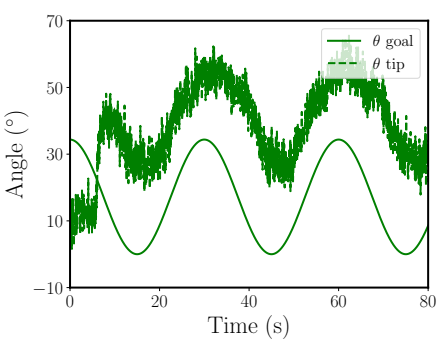

(b)

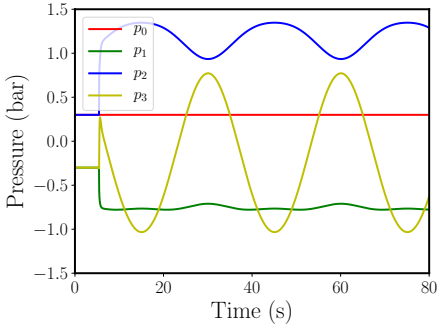

(c)

Fig. 12. The experimental results of the position and orientation control task for a robot with 3 bending segments $(N=3)$ show the plot of (a) the tip's (dashed lines) and goal position (solid lines), (b) the tip's (dashed lines) and goal orientation angle (solid lines), and (c) the input pressure values given to the main chamber (red line) and each bending pouches (green, blue, and yellow respectively).

interact with the environment. The use of integrated on-board sensors, such as bending sensors, to improve the estimation of the robot's states which in turns will improve the controller performance will also be investigated.

\section{REFERENCES}

[1] B. S. Homberg, R. K. Katzschmann, M. R. Dogar, and D. Rus, "Robust proprioceptive grasping with a soft robot hand," Autonomous Robots, vol. 43, no. 3, pp. 681-696, Mar. 2019. [Online]. Available: https://doi.org/10.1007/s10514-018-9754-1

[2] M. Cianchetti, T. Ranzani, G. Gerboni, T. Nanayakkara, K. Althoefer, P. Dasgupta, and A. Menciassi, "Soft robotics technologies to address shortcomings in today's minimally invasive surgery: the STIFF-FLOP approach," Soft robotics, vol. 1, no. 2, pp. 122-131, 2014.

[3] D. Rus and M. T. \& Tolley, "Design, fabrication and control of soft robots." Nature, vol. 521, pp. 467-475, 2015. [Online]. Available: http://www.nature.com/nature/journal/v521/n7553/abs/nature14543.html

[4] E. W. Hawkes, L. H. Blumenschein, J. D. Greer, and A. M Okamura, "A soft robot that navigates its environment through growth," Science Robotics, vol. 2, no. 8, 2017. [Online]. Available: http://robotics.sciencemag.org/content/2/8/eaan3028

[5] F. Putzu, T. Abrar, and K. Althoefer, "Plant-Inspired Soft Pneumatic Eversion Robot," in 2018 7th IEEE International Conference on Biomedical Robotics and Biomechatronics (Biorob), Aug. 2018, pp. 1327-1332.

[6] L. H. Blumenschein, L. T. Gan, J. A. Fan, A. M. Okamura, and E. W. Hawkes, "A Tip-Extending Soft Robot Enables Reconfigurable and Deployable Antennas," IEEE Robotics and Automation Letters, vol. 3, no. 2, pp. 949-956, Apr. 2018.

[7] N. Agharese, T. Cloyd, L. H. Blumenschein, M. Raitor, E. W. Hawkes, H. Culbertson, and A. M. Okamura, "HapWRAP: Soft Growing Wearable Haptic Device," in 2018 IEEE International Conference on Robotics and Automation (ICRA), May 2018, pp. 1-5.

[8] T. Abrar, F. Putzu, J. Konstantinova, and K. Althoefer, "EPAM: Eversive Pneumatic Artificial Muscle," in 2019 2nd IEEE International Conference on Soft Robotics (RoboSoft), Apr. 2019, pp. 19-24.

[9] A. Hassan, H. Godaba, and K. Althoefer, "Design Analysis of a Fabric Based Lightweight Robotic Gripper," in Towards Autonomous Robotic Systems, K. Althoefer, J. Konstantinova, and K. Zhang, Eds. Cham: Springer International Publishing, 2019, pp. 16-27.

[10] A. Stilli, E. Kolokotronis, J. Fra, A. Ataka, K. Althoefer, and H. A. Wurdemann, "Static Kinematics for an Antagonistically Actuated Robot Based on a Beam-Mechanics-Based Model," in 2018 IEEE/RSJ International Conference on Intelligent Robots and Systems (IROS), Oct. 2018, pp. 6959-6964.

[11] W. McMahan, B. A. Jones, and I. D. Walker, "Design and implementation of a multi-section continuum robot: Air-Octor," in 2005 IEEE/RSJ International Conference on Intelligent Robots and Systems, Aug. 2005, pp. 2578-2585.

[12] S. Sanan, P. S. Lynn, and S. T. Griffith, "Pneumatic Torsional Actuators for Inflatable Robots," Journal of Mechanisms and Robotics, vol. 6, no. 3, 2014. [Online]. Available: https://doi.org/10.1115/1.4026629

[13] M. M. Coad, L. H. Blumenschein, S. Cutler, J. A. R. Zepeda, N. D. Naclerio, H. El-Hussieny, U. Mehmood, J.-H. Ryu, E. W. Hawkes, and A. M. Okamura, "Vine Robots: Design, Teleoperation, and Deployment for Navigation and Exploration," CoRR, vol. abs/1903.00069, 2019. [Online]. Available: http://arxiv.org/abs/1903.00069
[14] F. Maghooa, A. Stilli, Y. Noh, K. Althoefer, and H. Wurdemann, "Tendon and pressure actuation for a bio-inspired manipulator based on an antagonistic principle," in Proc. IEEE Int. Conf. Robot. Autom., May 2015, pp. 2556-2561.

[15] S. M. H. Sadati, S. E. Naghibi, A. Shiva, I. D. Walker, K. Althoefer, and T. Nanayakkara, "Mechanics of Continuum Manipulators, a Comparative Study of Five Methods with Experiments," in Towards Autonomous Robotic Systems, Y. Gao, S. Fallah, Y. Jin, and C. Lekakou, Eds. Cham: Springer International Publishing, 2017, pp. 686-702.

[16] T. George Thuruthel, Y. Ansari, E. Falotico, and C. Laschi, "Control strategies for soft robotic manipulators: A survey," Soft robotics, vol. 5, no. 2, pp. 149-163, 2018.

[17] R. J. Roesthuis and S. Misra, "Steering of Multisegment Continuum Manipulators Using Rigid-Link Modeling and FBG-Based Shape Sensing," IEEE Transactions on Robotics, vol. 32, no. 2, pp. 372-382, Apr. 2016.

[18] A. Ataka, P. Qi, H. Liu, and K. Althoefer, "Real-time planner for multisegment continuum manipulator in dynamic environments," in Proc. IEEE Int. Conf. Robot. Autom., May 2016, pp. 4080-4085.

[19] A. Ataka, A. Shiva, H. K. Lam, and K. Althoefer, "Magnetic-FieldInspired Navigation for Soft Continuum Manipulator," in 2018 IEEE/RSJ International Conference on Intelligent Robots and Systems (IROS), Oct. 2018, pp. 168-173.

[20] J. D. Greer, L. H. Blumenschein, A. M. Okamura, and E. W. Hawkes, "Obstacle-Aided Navigation of a Soft Growing Robot," in 2018 IEEE International Conference on Robotics and Automation (ICRA), May 2018, pp. $1-8$.

[21] J. D. Greer, T. K. Morimoto, A. M. Okamura, and E. W. Hawkes, "A soft, steerable continuum robot that grows via tip extension," Soft robotics, vol. 6, no. 1, pp. 95-108, 2019.

[22] Y. Ansari, M. Manti, E. Falotico, M. Cianchetti, and C. Laschi, "Multiobjective Optimization for Stiffness and Position Control in a Soft Robot Arm Module," IEEE Robotics and Automation Letters, vol. 3, no. 1, pp. 108-115, Jan. 2018.

[23] A. Ataka, A. Stilli, J. Konstantinova, H. A. Wurdemann, and K. Althoefer, "Kinematic Control and Obstacle Avoidance for Soft Inflatable Manipulator," in Towards Autonomous Robotic Systems, K. Althoefer, J. Konstantinova, and K. Zhang, Eds. Cham: Springer International Publishing, 2019, pp. 52-64.

[24] R. Niiyama, X. Sun, C. Sung, B. An, D. Rus, and S. Kim, "Pouch motors: Printable soft actuators integrated with computational design," Soft Robotics, vol. 2, no. 2, pp. 59-70, 2015.

[25] H. Godaba, F. Putzu, T. Abrar, J. Konstantinova, and K. Althoefer, "Payload Capabilities and Operational Limits of Eversion Robots," in Towards Autonomous Robotic Systems, K. Althoefer, J. Konstantinova, and K. Zhang, Eds. Cham: Springer International Publishing, 2019, pp. 383-394.

[26] C. Wielgosz and others, "Bending and buckling of inflatable beams: some new theoretical results," Thin-walled structures, vol. 43, no. 8, pp. $1166-1187,2005$

[27] F. Bullo and R. M. Murray, "Proportional derivative (PD) control on the euclidean group," in Proc. European Control Conference, 1995, pp. 1091-1097.

[28] B. Siciliano, "Kinematic control of redundant robot manipulators: A tutorial," J. Intell. Robot. Syst., vol. 3, no. 3, pp. 201-212, 1990. [Online]. Available: http://dx.doi.org/10.1007/BF00126069 